\title{
REVIEW ARTICLE OPEN Evolution of therapy for limited stage diffuse large B-cell lymphoma
}

\author{
Alexandra E. Rojek ${ }^{1}$ and Sonali M. Smith $\mathbb{D}^{2 \bowtie}$ \\ (c) The Author(s) 2022
}

Diffuse large B-cell lymphoma (DLBCL) is the most common non-Hodgkin lymphoma (NHL), with limited-stage DLBCL defined as stage I or II disease. Risk stratification, initial treatment options, and relapse patterns are distinct from advanced-stage DLBCL, but there is limited data on the impact of biologic features on outcome. Patients have excellent outcomes, with $\sim 90 \%$ survival at 2 years. Over the past several years, sequential prospective trials and large registry studies have evaluated the optimal number of chemotherapy cycles and implemented PET-adapted approaches to reduce the need for radiotherapy. Special consideration must still be given to cases of bulky disease, extranodal disease, fully resected scenarios, and adverse biologic features such as highgrade B-cell lymphoma with double/triple hit rearrangements. This review presents the evolution of a modern management approach, with a discussion of recent treatment-defining studies.

Blood Cancer Journal (2022)12:33; https://doi.org/10.1038/s41408-021-00596-z

\section{INTRODUCTION AND HISTORICAL PERSPECTIVE}

Diffuse large B-cell lymphoma (DLBCL) is the most common nonHodgkin lymphoma (NHL), representing almost one-quarter of all new NHL cases per year in the United States [1]. It is biologically heterogeneous; historically, limited-stage disease was defined as Ann Arbor stage I or II disease with sites that could be encompassed in a single radiation field. Currently, limited or early-stage disease is defined as stage I or II, and advanced-stage disease is defined as stage III or IV by Lugano criteria [2]. Bulky disease, which is variably defined in the literature, is an important modifier to classical staging and is discussed further below. Large descriptive studies report that the median age of patients with the limited-stage disease is in the sixth decade of life, with a slight male predominance; [3-6], the most common anatomic sites of disease are in cervical lymph nodes and/or head and neck region, including Waldeyer's ring. There remains debate as to whether the delineation between limited and advanced stage reflects earlier identification of a disease or a biologically distinct entity with different risks and outcomes features.

The modern approach to the treatment of limited-stage DLBCL was influenced by SWOG 8736. This phase 3 trial, conducted in the pre-rituximab era (1988-1995), established combined modality therapy as a standard of care in the pre-rituximab era. SWOG S8736 showed that three cycles of cyclophosphamide, doxorubicin, vincristine, and prednisone (CHOP) with radiation therapy (RT) were non-inferior to eight cycles of $\mathrm{CHOP}$ alone. This defined a new standard treatment length and inclusion of radiation for limited-stage patients [7] which has continued to serve as the foundation for subsequent dedicated limited-stage disease trials.

SWOG S8736 also developed a risk stratification scoring system in limited-stage DLBCL that retains utility in the modern era. The International Prognostic Index (IPI) for DLBCL was adjusted to better stratify prognosis in limited-stage disease by removing the number of extranodal sites and dichotomizing stage as I versus II. This stagemodified IPI (smIPI) thus includes one point each for age $>60$ years, stage II disease, elevated serum lactate dehydrogenase (LDH), and performance status of two or above $[7,8]$. In the pre-rituximab era, those with no risk factors, and thus stage I disease, had the best outcomes with 5-year overall survival (OS) of 95\%; for those with one to two risk factors, 5 -year OS dropped to $77 \%$, and $50 \%$ for three or more risk factors $[7,9]$. This smIPI model was more powerful than an age-adjusted risk stratification alone [10]. In the rituximab era, outcomes in each IPI risk group have improved across both limited and advanced disease groups, and smIPI retains utility [11, 12].

The anti-CD20 monoclonal antibody, rituximab, improves survival in limited-stage disease just as it does for advancedstage DLBCL $[13,14]$. The MabThera International Trial (MInT) study found that non-bulky, limited-stage disease treated with R-CHOP had better 6-year event-free survival (EFS) at $84.3 \%$ and 6 -year OS of $94.9 \%$ than CHOP alone. Notably, these survival benefits occurred without a significant increase in toxicity or rate of secondary hematologic malignancy in long-term follow-up $[15,16]$. This study helped establish the superiority of chemoimmunotherapy in younger patients with a good prognosis, limitedstage DLBCL.

The treatment of limited-stage DLBCL patients in the modern era has evolved to reconsider the role of RT, the optimal length of systemic therapy, and the emerging role of metabolic imaging via positron emission tomography (PET) in response-adapted management. This review will focus on therapies and studies focusing on limited-stage DLBCL (Table 1), the inherent challenges and future considerations for this disease, and our recommended approach to these patients as diagnostics and therapeutics continue to evolve in the modern era.

\footnotetext{
${ }^{1}$ Department of Medicine, University of Chicago, Chicago, IL, USA. ${ }^{2}$ Section of Hematology/Oncology, Department of Medicine, University of Chicago, Chicago, IL, USA

email: smsmith@medicine.bsd.uchicago.edu
}

Received: 19 July 2021 Revised: 4 November 2021 Accepted: 24 November 2021

Published online: 24 February 2022 


\section{IS THE BIOLOGY OF LIMITED-STAGE DLBCL DISTINCT FROM} ADVANCED STAGE?

DLBCL is increasingly understood to be a highly heterogeneous neoplasm with many variations in morphology, gene expression, and biological regulation. The biology of limited-stage DLBCL versus advanced-stage disease is not clearly understood, and it remains to be determined whether it is a manifestation of earlier disease presentation or truly biologically distinct. In advancedstage disease, known prognostic indicators include cell-of-origin (COO) and deregulation of MYC and BCL2 via rearrangements or protein overexpression. In advanced-stage disease, COO may impact treatment selection [17-20], with GCB cases having favorable prognoses over non-GCB cases [21, 22]. Deregulation of $M Y C$ and $B C L 2$ and/or BCL6 through translocations results in "high-grade B-cell lymphoma with double-hit/triple hit (HGBL$\mathrm{DHL} / \mathrm{THL})^{\prime \prime}$ and is associated with inferior survival outcomes $[23,24]$. Overexpression of the associated proteins, also a negative prognostic indicator in advanced-stage disease, is often dubbed as double-expressor lymphoma (DEL) $[25,26]$.

Although data regarding $\mathrm{COO}$ in limited-stage cases is more scarce, $\sim 60-75 \%$ are thought to have GCB origin $[27,28]$. The prognostic significance of $\mathrm{COO}$ among limited-stage diseases probably parallels advanced-stage disease, although COO breakdown based on stage is scarcely described. In one study, despite similar proportions of limited-stage disease within GCB and nonGCB subtypes, those with non-GCB cell-of-origin fared worse with a 5 -year progression-free survival (PFS) of $48 \%$ and OS of $56 \%$, compared to 73 and $78 \%$ for GCB cases [29], although this was across all stages of DLBCL. In the S1001 trial discussed further below, GCB cases had a favorable PFS over non-GCB subtypes [4].

The prevalence of limited-stage disease in $\mathrm{DHL}$ or $\mathrm{DEL}$ populations is variable, and also incompletely studied to date. In a study of DHL patients achieving first complete remission, $24 \%$ had limited-stage disease [30]; another study of limited-stage disease found only $19 \%$ DEL cases and 4\% DHL [27]. The impact of DHL or DEL status on prognosis in limited-stage disease has not been directly addressed, but subgroup analyses have found no difference in outcomes for DHL/DEL populations [4, 27, 31].

Another histopathologic variation of DLBCL is a preceding or concurrent indolent disease component. In a recent prospective cohort study of all DLBCL stages, one-third of limited-stage cases had a concurrent follicular lymphoma [32]. COO for these cases of concurrent indolent lymphoma was most often $G C B$, and outcomes were comparable to de novo GCB-DLBCL, across stages. Many clinical trials discussed below exclude cases of transformed indolent lymphoma, or patients who had received prior therapy; as such, the true incidence and significance of transformed indolent disease to limited-stage DLBCL remain unknown.

With much unknown as to the significance of these prognostic biomarkers in limited-stage $D L B C L$, there is an unmet need and opportunity for future research to better characterize the biology of limited-stage DLBCL, particularly as genomic studies to date [33] have not separated limited-stage from advanced-stage disease - leaving the question of whether limited-stage DLBCL is a biologically distinct entity, entirely unanswered.

\section{INITIAL TREATMENT APPROACH: COMBINED MODALITY OR CHEMOTHERAPY ALONE?}

Similar to advanced-stage DLBCL, optimal regimens for limitedstage DLBCL evolved to include rituximab with chemotherapy regimens, followed by the incorporation of PET in initial staging and response assessment. These two landmark changes have influenced contemporary trial design and standard therapies, as the inclusion of RT in treatment of limited-stage DLBCL has continued to be debated. Radiation alone is insufficient as first-line therapy, with high rates of recurrence outside the field of radiation $[34,35]$, and radiation (when used) is usually part of a combined 
modality approach. Below we discuss significant studies which address the role of RT in the modern treatment era.

\section{Studies in the pre-chemoimmunotherapy and pre-PET era}

In the pre-rituximab era, a number of studies evaluated combined modality therapy versus chemotherapy alone, with conflicting results. As discussed above, the SWOG 8736 study initially established that three cycles of CHOP plus RT has improved 5 -year outcomes [7]. However, the modern application of this trial is limited by a comparator arm that included more chemotherapy cycles than are standard today. In addition, with prolonged followup nearing 18 years, there were no statistically significant differences in the PFS or OS of these groups, and there was a notable pattern of continuous relapse in both arms [36]. Two pivotal Groupe d'Etude des Lymphomes de l'Adulte (GELA) trials in the early 1990s suggested excellent outcomes for chemotherapy alone. In the GELA-93-1 study, patients with limited disease and no adverse IPI prognostic factors were randomized to treatment with CHOP and RT or chemotherapy alone with doxorubicin, cyclophosphamide, vincristine, bleomycin, and prednisone (ACVBP) with sequential consolidation. They found improved 5 -year EFS and OS for those receiving ACVBP alone than R-CHOP with RT at a dose of 40 Gy [37]. In comparing different chemotherapy regimens, however, the dose intensity of three cycles of ACVBP was estimated to be about $150 \%$ of an equivalent three cycles of CHOP. The GELA-93-4 study found no difference in outcomes of patients with limited-stage disease over the age of 60 treated with four cycles of CHOP alone or four cycles of $\mathrm{CHOP}$ with RT [38]. There was, however, a heightened risk of secondary malignancy in the radiation arm. An additional trial by the Eastern Cooperative Oncology Group (ECOG) 1484 also showed no benefit for RT in OS after eight cycles of CHOP [6]. As expected, RT provided excellent local control; among refractory patients in this study - only 3 out of 17 patients from the RT arm progressed at original disease sites, compared with 15 out of 31 patients in the chemotherapy-alone arm.

It is important to note that patient populations differed in these trials, which further confounds interpretation. In particular, the inclusion of patients with bulky disease, variable smIPI risk scores, and other features varied, limiting the ability to directly compare the recommendations and conclusions of each study.

\section{Studies with chemoimmunotherapy in the pre-PET era}

The introduction of rituximab improved survival across all stages of DLBCL, including limited-stage disease. Prior to the introduction of PET as a staging and response assessment marker, prospective trials evaluating the role of RT had conflicting results, at least partially based on the inclusion of bulky disease, further examined below.

Building upon S8736, SWOG 0014 added rituximab to the three cycles of CHOP with RT at a dose of 40-46 Gy. The study excluded bulky disease, defined as $>10 \mathrm{~cm}$, and required patients to have at least one smIPI adverse risk factor. This phase 2 multicenter study demonstrated a 2-year PFS of 93\% and 4-year PFS of 88\%; 2-year OS was $95 \%$ and 4 -year OS was $92 \%$. When compared indirectly to S8736, this was an improvement on both 4-year PFS of $78 \%$ and 4 -year OS of $88 \%$ [39]. Despite including an older population with more adverse risk factors, S0014 suggested improved outcomes with chemoimmunotherapy.

Among patients receiving rituximab along with chemotherapy, what is the impact of consolidative RT? A retrospective study from MD Anderson looked at the impact of RT, and concluded that among all stages of DLBCL treated in the rituximab era $(64 \%$ of limited-stage patients received 6-8 cycles of R-CHOP), RT improved PFS and OS [40]. Among limited-stage cases, those receiving RT had a 5 -year OS of $92 \%$ and a 5 -year PFS of $82 \%$, compared to 73 and $68 \%$, respectively for those not receiving RT. Notably, however, bulky disease in this study was defined as
$>5 \mathrm{~cm}$, and a separate analysis of bulky disease with larger size definitions was not conducted.

The prospective study by LYSA and Groupe Ouest-Est des Leucémies et des Autres Maladies du Sang (GOELAMS), LYSA/ GOELAMS 02-03, assessed 334 patients with non-bulky limitedstage DLBCL, randomized to R-CHOP with or without RT. Patients with bulky disease defined as $>7 \mathrm{~cm}$ were excluded, but all smIPI risk scores were included. All patients were randomized to receive $\mathrm{RT}$ at a dose of $40 \mathrm{~Gy}$ or not, a minimum of four cycles of R-CHOP, and those with smIPI $>1$ received an additional two cycles of R-CHOP. After four cycles of R-CHOP, all patients had a PET response assessment, and those in a complete remission (CR) continued treatment whereas those with a partial response (PR) received an additional two cycles of R-CHOP regardless of which treatment arm they were enrolled in. There was no statistically significant difference in 5-year EFS at $89 \%$ in the R-CHOP alone arm versus $92 \%$ in the R-CHOP plus RT arm. Additionally, 5-year OS was not significantly different at $92 \%$ for $\mathrm{R}-\mathrm{CHOP}$ alone versus $96 \%$ for R-CHOP plus RT [5]. This trial supports excellent outcomes without RT in non-bulky limited-stage DLBCL, including those with higher smIPI scores.

The UNFOLDER trial was designed as a $2 \times 2$ trial to assess the benefit of the R-CHOP treatment schedule and inclusion of RT at a dose of $40 \mathrm{~Gy}$. However, as is further discussed in the section dedicated to bulky disease, the trial was stopped early due to excessive events in the arm without RT. There was, however, no difference in PFS or OS between groups receiving RT or not, including when restricted to those with bulky disease [41].

Although the retrospective and smaller studies present contradictory evidence in the rituximab era of the added benefit of RT, the LYSA/GOELAMS 02-03 trial suggests that for patients with nonbulky disease, RT can be omitted without affecting prognosis.

\section{Studies in the chemoimmunotherapy and PET era}

The introduction of PET as a standard imaging modality in lymphoma introduced another variable in management decisions for limited stage DLBCL, with three key areas of impact: staging, end-of-treatment (EOT) assessment for prognosis, and responseadapted investigations. The use of PET for staging often results in "up-staging", where it is able to detect additional sites of disease in $35 \%$ of patients, with $12 \%$ resulting in higher staging [42]. One retrospective study showed that EOT scans had a positive predictive value (PPV) of $56 \%$ among those with IPI $<3$, compared with $80 \%$ for those with $I P I \geq 3$. When used to monitor for relapse, PET has a $95 \%$ sensitivity and $97 \%$ specificity [43]. Overall, a negative PET scan at EOT portends an excellent prognosis, and surveillance CT imaging beyond 2 years is not recommended [44].

Several prospective trials have evaluated PET-adapted approaches in limited-stage DLBCL, with the goal of limiting both chemotherapy and RT. S1001 is a US Intergroup study prospectively assessing the role of interim PET (iPET) scans after three cycles of R-CHOP in non-bulky disease. Those with a negative iPET, defined as Deauville score three or less, received one additional cycle of R-CHOP whereas those with a positive iPET received 36 Gy of RT followed by ibritumomab tiuxetan radioimmunotherapy with rituximab. One hundred and twenty-eight patients were included in this study, with a median age of 62 years, stage I disease in $62 \%, 14 \%$ with elevated $\mathrm{LDH}, 66 \%$ with head and neckonly involvement, and $43 \%$ with extranodal involvement. smIPI was zero in $27 \%$, one in $42 \%$, two in $28 \%$, and three in $4 \%$ of included patients. Of these 128 patients, 110 had a negative iPET and did not receive RT. There was no statistically significant difference in the 5 -year PFS of $86 \%$ for iPET-positive patients and $89 \%$ for iPET-negative patients; likewise, there was no statistical difference in the 5-year OS of $85 \%$ for iPET-positive patients and $91 \%$ for iPET-negative patients. Only six of 132 patients relapsed at a median 4.9 years follow-up time [4]. This PET-adapted approach successfully reduced the number of chemoimmunotherapy cycles 
and the need for radiation with equivalent and excellent outcomes.

The British Columbia Cancer Agency (BCCA) adopted a PETbased approach where patients achieving a CR by PET after three cycles of R-CHOP were treated with one additional cycle of RCHOP, while patients with evidence of residual disease-defined by either International Harmonization Project criteria or minimum Deauville score three-also received RT. Eighty percent of the study population had at least one smIPI risk factor, with a 3-year PFS of $92 \%$ and 3 -year OS of $96 \%$ for those with a negative iPET, compared to 60 and $83 \%$, respectively, for those with positive iPET $[45,46]$. This regional analysis suggests that a response-based assessment using iPET holds promise in allowing for a reduction in chemoimmunotherapy length and associated toxicity, whereas there remains a need for treatment optimization for patients with positive iPET.

The recent LYSA group study, LNH 2009-1B, further evaluated the role of early PET imaging in assessing whether four cycles of $\mathrm{R}$-CHOP were non-inferior to six cycles. In the experimental arm, those who had a negative iPET after two cycles received a total of four cycles whereas those with a positive iPET after two cycles received a total of six cycles. Early results showed that with a median follow-up of 5 years, the 3-year PFS was $89 \%$ in the standard arm where all patients received six cycles of R-CHOP, and $92 \%$ in the experimental arm [47]. The non-inferiority of this PETadapted approach allowing for abbreviated chemoimmunotherapy in those with a negative iPET further suggests that excellent outcomes can be achieved in this population with less treatment.

Similarly, the ongoing OPTIMAL $>60$ aims to use iPET to evaluate response after two cycles of therapy, with either additional two cycles of therapy for those with a negative iPET or additional cycles with RT for those with a positive iPET. This study also explores the role of other PET-derived biomarkers such as metabolic tumor volume, which have been shown in other studies to be predictive of PFS [48] and OS [49].

\section{HOW MUCH CHEMOIMMUNOTHERAPY IS ENOUGH?}

Historically, the number of chemotherapy cycles was only abbreviated when adding RT, and studies without RT often delivered more cumulative cytotoxic chemotherapy [7, 36, 37]. However, can chemotherapy cycles be reduced concurrently with omission or reduction of RT? Overall survival in several studies exceeds $90 \%$, raising the possibility of overtreatment for patients at lowest risk.

The randomized FLYER trial addressed whether, among limitedstage DLBCL patients, four cycles of R-CHOP alone were noninferior to six cycles of R-CHOP. The trial enrolled 588 patients with non-bulky disease and no adverse IPI risk factors, a median age of 48 years, $32 \%$ extranodal involvement, and $40 \%$ of patients with stage II disease. This study showed that four cycles of R-CHOP were non-inferior to six, with a 3-year PFS of $96 \%$ for those who received four cycles, and $94 \%$ for six cycles. Likewise, the 3-year OS was $99 \%$ for those with four cycles, and $98 \%$ for six cycles. Fewer adverse events, including cytopenias and non-hematologic events, occurred in the four-cycle group, as expected with a smaller cumulative dose of chemotherapy [50]. This trial did include two additional rituximab infusions as consolidation, but prior meta-analyses have not shown survival benefit for rituximab consolidation [14, 51, 52]. An added consideration is the ongoing COVID-19 pandemic, where fewer rituximab doses might be advantageous in terms of infectious risk and response to available vaccines; several recent trials suggest that mounting a sufficient immune response is delayed for 12 months after rituximab exposure.

It is important to note that the FLYER trial was limited to patients without risk factors; extrapolating these findings to other groups may not have the same outcomes. Nevertheless, this trial sets a treatment standard for young patients without risk factors and spares higher doses of cumulative chemoimmunotherapy.

\section{SPECIAL MANAGEMENT CONSIDERATIONS IN LIMITED-STAGE DISEASE}

As modern treatment approaches to limited-stage DLBCL have evolved to shorten courses of chemoimmunotherapy and potentially omit RT when incorporating PET-based response assessments, the need to consider special populations that may require modified treatment approaches remains. There is minimal data guiding treatment in the very elderly, but our approach is to select R-CHOP, R-mini-CHOP, or R-CEOP akin to approaches in advanced-stage disease, but limit the number of cycles similar to approaches in limited-stage disease for younger patients. The cases of bulky disease, DHL and DEL, extranodal involvement, CNS prophylaxis, and fully resected disease are discussed below.

\section{Bulky disease}

Bulky disease is variably defined within limited-stage DLBCL; most limited-stage studies exclude stage II bulky disease, and bulky stage I disease is a relatively rare entity. Other studies consider bulky stage II disease akin to advanced stage DLBCL, although cutoffs for defining bulk vary. Studies such as FLYER, LYSA/ GOELAMS 02-03, SWOG 0014, and S1001 discussed above all excluded patients with bulky disease and, as such, many of their conclusions must be applied with caution for patients with bulky disease.

The UNFOLDER trial randomized patients with the bulky disease to chemoimmunotherapy with or without RT, where bulky disease was defined as $>7.5 \mathrm{~cm}$, and extranodal disease was included. The study was stopped early because of excessive failures based on pre-defined criteria in the non-RT arm among patients with bulky disease [41]. These events were attributed to partial responses requiring localized RT; however, there was no difference in PFS or OS (3-year PFS of $89 \%$ vs $81 \%$; 3 -year OS of $93 \%$ each) between groups receiving RT or not. Although retrospective, a different analysis from MD Anderson included 190 patients who had limited stage disease; $54 \%$ received RT, and $48 \%$ of those had bulky disease (defined as $>5 \mathrm{~cm}$ in diameter). They did not find that bulky disease, across all stages, was associated with worse outcomes [40].

Most of these studies have been limited in their ability to definitively address the question of bulky limited-stage disease in part because of sample size of this sub-population. A recent retrospective study in Finland aimed at answering this outstanding question with the adjunctive use of iPET. One hundred twenty-three all-stage DLBCL patients had bulky disease and $44 \%$ of these received RT. Among limited-stage patients, the presence of bulky tumor was associated with inferior prognosis, with 2-year PFS of $53 \%$ compared to $90 \%$ to those with non-bulky disease; however, the benefit of RT in delaying time to progression disappeared after excluding primary refractory cases. They additionally noted that within bulky disease, a negative iPET retained its favorable prognostic benefit, with a 2-year PFS of $87 \%$ for those with negative iPET and bulky disease, compared to $57 \%$ for those with a positive iPET [53]. While retrospective in nature, this study suggests that RT provides an additional benefit in cases of bulky limited-stage disease such as primary refractory disease, but reinforces that a negative iPET retains its prognostic power even in the presence of other risk factors and that RT may not be necessary.

\section{Double-hit lymphoma and dual expression of MYC/BCL2}

The prognostic significance of $\mathrm{DHL}$ and $\mathrm{DEL}$ within limited-stage disease is incompletely characterized. One prior retrospective study assessing DHL patients suggested that low-risk DHL patients may benefit from RT in prolonging time to relapse [54]. However, a 
recent retrospective study [3], focusing on limited-stage MYCrearranged cases showed that, among 104 patients, the overall response rate was $91 \%$ to chemoimmunotherapy. The CR rate for those with DHL was $75 \%$ compared to $98 \%$ for MYC-only rearrangements. The 2-year PFS and OS were 78 and $86 \%$ for the entire cohort, and did not differ between those receiving RT or among DHL patients. Additionally, for limited-stage patients, there was no demonstrated benefit in using intensive chemoimmunotherapy over R-CHOP for either MYC-only rearranged cases or for DHL. Altogether these studies suggest that DHL or DEL cases of limited-stage disease may have a different prognosis than what is expected in advanced-stage disease, although numbers are relatively small. While awaiting further studies to characterize these differences, there is no clear role for intensified treatment for these populations at present.

\section{Extranodal involvement}

Another special population to consider in limited-stage disease is extranodal disease, which has been associated with worse outcomes than nodal disease in retrospective studies in the rituximab era [55], but with conflicting results in the limited-stage population. Approximately $50 \%$ of extranodal DLBCL cases present as stage I, [56] posing a unique challenge in management compared to advanced-stage disease. Additionally, the smIPI, unlike IPI, does not consider multiple extranodal sites of disease a risk factor.

Of the studies discussed above, the LYSA/GOELAMS 02-03 study which assessed the role of RT with 4-6 cycles of R-CHOP and included patients with extranodal disease, did not find that the presence of extranodal sites impacted EFS or OS [5]. The S1001 trial similarly did not find worse outcomes for extranodal involvement [4]. Other studies such as FLYER excluded patients with extranodal disease, and MInT allowed clinicians to use RT at their discretion, but did not separately analyze extranodal disease $[15,16]$.

A recent retrospective study of stage I DLBCL patients [55], however, suggested that extranodal disease is associated with inferior outcomes. Among 341 stage I cases, $66 \%$ had extranodal involvement, with the most common sites being bone, stomach, testis, intestine, and breast. $69 \%$ of these extranodal patients received RT, compared to $68 \%$ without extranodal involvement. Patients with extranodal disease had worse outcomes, with a 10 year OS of $70 \%$ and 10 -year PFS of $63 \%$, compared to 89 and $85 \%$ for nodal disease. Consolidative RT was associated with improved OS and PFS within extranodal disease. Those with a positive EOT PET scan did not have inferior survival, but $75 \%$ of these patients received consolidative RT; there was no added benefit from RT for those with a negative EOT PET. All relapses occurred outside the radiation field, and the most common sites were nodal and central nervous system (CNS). Only $8 \%$ of patients with extranodal disease relapsed, and of those who did, 30\% relapsed at the original disease site. Patients who relapsed after RT all presented with distant sites of relapse. Those with CNS relapse had initial involvement of breast and testes, suggesting that CNS prophylaxis for those with breast involvement may be of benefit. In contrast, there is an ongoing SWOG analysis that shows no adverse impact of extranodal presentation. This retrospective analysis included patients with non-bulky disease from S1001 as well as S0313 and S0014, and found that there was no significant difference in either EFS or OS at 10 years based on whether patients had extranodal or only nodal disease [57].

Another retrospective study of 126 stage I DLBCL patients with extranodal involvement [58] analyzed patterns of relapse, with the most common initial sites including gastrointestinal, bone, and nasopharyngeal disease. Consistent with the above study, they showed a $15 \%$ relapse rate, with $79 \%$ having distant sites of relapse and $25 \%$ being $>5$ years out from initial diagnosis. Additionally, $32 \%$ of relapses involved the CNS, all among cases initially involving the testes (despite prophylaxis), nasopharynx, and nodal disease.

Specifically for limited stage testicular disease, although rareoccurring in $\sim 1 \%$ of extranodal cases [59] - a combination of R$\mathrm{CHOP}$, intrathecal prophylaxis with methotrexate (discussed below), and contralateral testicular radiation of 30Gy in stage I and II patients resulted in a 5-year PFS of 74\% and 5-year OS of $85 \%$ [60]. This combination of chemoimmunotherapy with radiation and CNS prophylaxis is thus highly effective.

Although further prospective trial data is needed to determine the role of additional therapy for extranodal disease, the inclusion of radiation is controversial. The studies above suggest that by incorporating EOT PET, such response-based assessment may be able to better identify extranodal disease which would benefit from consolidative RT. Additionally, certain presentations of extranodal disease may merit a consideration of CNS prophylaxis, discussed below.

\section{CNS prophylaxis}

There is no data guiding the indication and/or utility of CNS prophylaxis in limited-stage disease. When evaluating the CNS-IPI, features most associated with the risk of CNS relapse that is pertinent in limited-stage disease are extranodal involvement or involvement at a site considered "high risk" such as nasal sinuses [61]. Studies evaluating the CNS relapse risk in limited-stage disease suggest a higher risk of relapse if there was an MYC rearrangement; there was no impact of primary disease site [58]. Among relapses, one-third had a CNS relapse. A recent retrospective review of CNS prophylaxis and relapses in all stages of DLBCL found an increased risk of testicular CNS relapse, but no difference in CNS relapse rates between routes of prophylaxiseither systemic high-dose methotrexate (HD-MTX) or intrathecal methotrexate (IT-MTX) [62]. With this lack of evidence of a difference in relapse rates, IT-MTX is often a preferable option for prophylaxis given lower rates of toxicity and delay in systemic therapy.

National Comprehensive Care Network (NCCN) guidelines for CNS prophylaxis, although not specific to limited stage disease, include high-risk disease sites as an indication for prophylaxis, including kidney, adrenal, testicular, and breast [1]. Other retrospective work has suggested that involvement of sites such as paranasal sinuses, breasts, and testes should also receive CNS prophylaxis [63, 64]. Overall, there is insufficient data to definitively guide decision-making regarding CNS prophylaxis, and more data is needed.

\section{Fully resected disease}

In some cases of limited-stage disease, the entirety of the tumor may be resected during the diagnostic biopsy, or in other special cases such as obstruction from intestinal extranodal involvement [65]. Traditionally, these patients were given the same duration of therapy as other limited-stage disease patients. Subgroup analyses of existing studies have attempted to better answer whether fully resected disease allows for abbreviated therapy, although this varies by initial site of disease and other considerations.

A phase 2 study conducted by the Consortium for Improving Survival of Lymphoma (CISL), CISL 12-09, set out to determine the safety and efficacy of an abbreviated three cycles of R-CHOP after total resection of limited stage disease. At a median follow-up time of 39.5 months, only one patient out of 22 progressed, with an estimated 2-year OS was 95\% [66]. In extended follow-up at 5 years, no additional events related to disease progression or death occurred [67]. Although limited by study size, this supports a limited systemic treatment course for patients with fully resected limited stage DLBCL.

Analysis of patients enrolled in the Positron Emission Tomography-guided Therapy of Aggressive non-Hodgkin Lymphomas (PETAL) trial [68] found that 52 patients had fully resected 

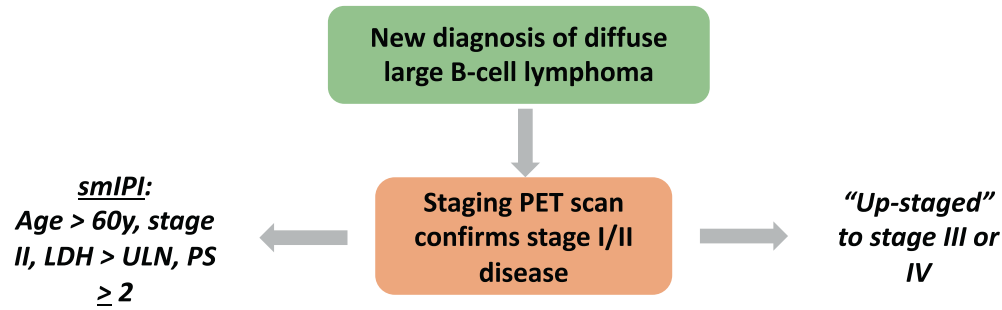

$\geq 2$

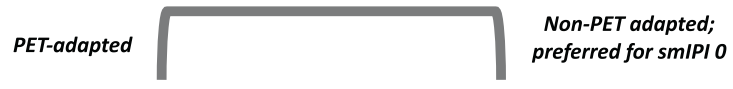

R-CHOP $\times 3$

R-CHOP x 4

iPET
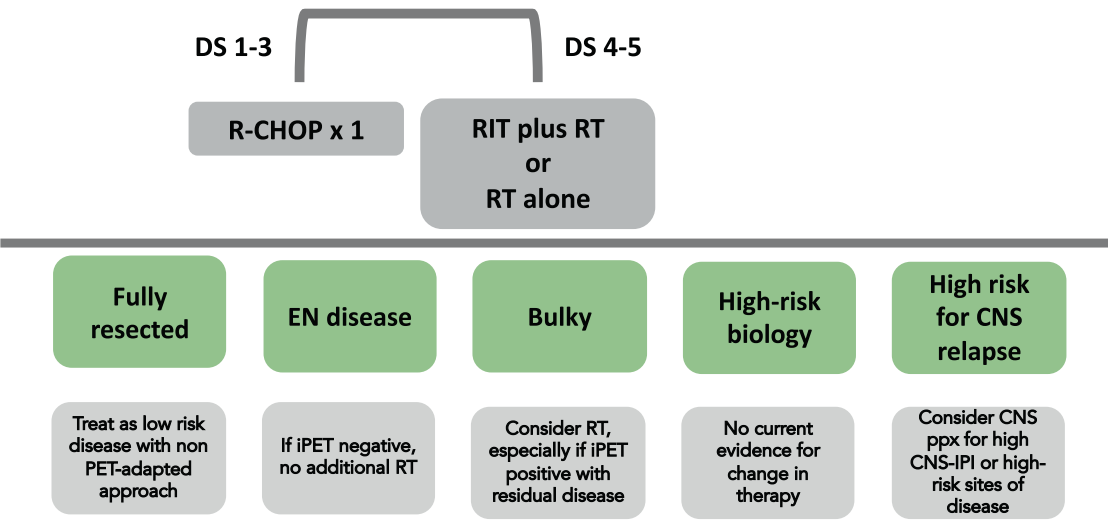

Fig. 1 Our approach to the management of limited stage diffuse large B-cell lymphoma (DLBCL). Abbreviations: iPET interim PET, smIPI stage modified IPI, RIT radioimmunotherapy, DS Deauville score, ppx prophylaxis, PS performance status, ULN upper limit normal.

stage I disease by baseline PET, with most patients receiving 6 cycles of R-CHOP. Those with surgically resected disease under age 60 had an improved 2-year PFS and OS of 100\% compared to 92 and $95 \%$, respectively, for those with incompletely resected disease; there were no statistically significant differences between groups for those over age 60 [65]. Furthermore, given the results of the FLYER trial above, it is reasonable to extrapolate that four cycles of chemoimmunotherapy would be sufficient.

A retrospective study of 250 primarily intestinal, limited-stage patients receiving six cycles of $\mathrm{CHOP}$ or $\mathrm{R}-\mathrm{CHOP}$ showed a significantly better $\mathrm{CR}$ rate in the combined surgery and chemotherapy group of $85 \%$ compared to $64 \%$ for those receiving chemotherapy alone, who also had local relapses more frequently. Of those who underwent surgery, $60 \%$ were for mass resection and $31 \%$ for obstruction. Surgically resected patients had a 3-year PFS of $82 \%$ and a 3 -year OS of $91 \%$, compared to 52 and $62 \%$ for chemotherapy alone. There was no survival benefit, however, in surgical resection of advanced-stage cases involving intestinal disease [69]. Importantly, this finding suggests that while in advanced-stage disease surgical resection does not provide a survival benefit, in specific anatomical considerations of limitedstage disease, surgical resection may be useful.

\section{OUR APPROACH TO MANAGEMENT}

The current outcomes for patients with limited stage DLBCL are outstanding, and the field has evolved to limit both short- and longterm toxicities without compromising efficacy. Our approach (Fig. 1) is based on initial clinicopathologic features, incorporation of the smIPI, and the results of interim PET scanning. Patients with a low smIPI or negative iPET, including fully resected disease, can be treated with a maximum of four cycles of R-CHOP without consolidative radiotherapy. For those with a positive iPET, additional radiation and/or additional chemoimmunotherapy may hold a survival benefit. RT is also a consideration for those with bulky $(>7.5 \mathrm{~cm})$ or extranodal disease, with additional guidance for individual cases as discussed above. Such an approach minimizes the toxicity of therapy without sacrificing benefit of outcomes. Additional factors also need to be considered, such as CNS prophylaxis for high-risk disease sites such as testicular, breast, and nasopharyngeal involvement, and limited treatment courses for those with the fully resected disease at staging.

Upcoming studies in limited stage DLBCL will aim to further refine the role of earlier iPET in reducing the need for further therapy. Additionally, further insight into the specific disease biology of limited DLBCL should be pursued to further our understanding of new DLBCL classification that may not be dependent on stage alone. This may change the diagnosis and management of limited-stage DLBCL and has the potential to even further improve outcomes and toxicities in a disease that has seen great strides into the modern treatment era.

\section{REFERENCES}

1. Liu Y, Barta SK. Diffuse large B-cell lymphoma: 2019 update on diagnosis, risk stratification, and treatment. Am J Hematol. 2019;94:604-16.

2. Cheson BD, Fisher RI, Barrington SF, Cavalli F, Schwartz LH, Zucca E, et al. Recommendations for initial evaluation, staging, and response assessment of Hodgkin and non-Hodgkin lymphoma: the Lugano classification. J Clin Oncol. 2014;32:3059-68.

3. Torka P, Kothari SK, Sundaram S, Li S, Medeiros LJ, Ayers EC, et al. Outcomes of patients with limited-stage aggressive large B-cell lymphoma with high-risk cytogenetics. Blood Adv. 2020;4:253-62.

4. Persky DO, Li H, Stephens DM, Park SI, Bartlett NL, Swinnen L, et al. Positron emission tomography-directed therapy for patients with limited-stage diffuse large B-cell lymphoma: results of intergroup national clinical trials network study S1001. J Clin Oncol. 2020;38:3003-11. 
5. Lamy T, Damaj G, Soubeyran P, Gyan E, Cartron G, Bouabdallah K, et al. R-CHOP 14 with or without radiotherapy in nonbulky limited-stage diffuse large B-cell lymphoma. Blood. 2018;131:174-81.

6. Horning SJ, Weller E, Kim K, Earle JD, O'Connell MJ, Habermann TM, et al. Chemotherapy with or without radiotherapy in limited-stage diffuse aggressive nonHodgkin's lymphoma: Eastern Cooperative Oncology Group study 1484. J Clin Oncol. 2004;22:3032-8.

7. Miller TP, Dahlberg S, Cassady JR, Adelstein DJ, Spier CM, Grogan TM, et al. Chemotherapy alone compared with chemotherapy plus radiotherapy for localized intermediate- and high-grade non-Hodgkin's lymphoma. N. Engl J Med. 1998:339:21-26.

8. Miller TP. The limits of limited stage lymphoma. J Clin Oncol 2004;22:2982-4.

9. Shenkier TN, Voss N, Fairey R, Gascoyne RD, Hoskins P, Klasa R, et al. Brief chemotherapy and involved-region irradiation for limited-stage diffuse large-cell lymphoma: an 18-year experience from the British Columbia Cancer Agency. J Clin Oncol. 2002;20:197-204.

10. Møller MB, Christensen BE, Pedersen NT. Prognosis of localized diffuse large B-cell lymphoma in younger patients. Cancer. 2003;98:516-21.

11. Ziepert M, Hasenclever D, Kuhnt E, Glass B, Schmitz N, Pfreundschuh M, et al. Standard International prognostic index remains a valid predictor of outcome for patients with aggressive CD20+ B-cell lymphoma in the rituximab era. J Clin Oncol. 2010;28:2373-80.

12. Sehn LH, Berry B, Chhanabhai M, Fitzgerald C, Gill K, Hoskins $P$, et al. The revised International Prognostic Index (R-IPI) is a better predictor of outcome than the standard IPI for patients with diffuse large B-cell lymphoma treated with R-CHOP. Blood. 2007;109:1857-61.

13. Coiffier B, Lepage E, Briere J, Herbrecht R, Tilly H, Bouabdallah R, et al. CHOP chemotherapy plus rituximab compared with $\mathrm{CHOP}$ alone in elderly patients with diffuse large-B-cell lymphoma. N. Engl J Med. 2002;346:235-42.

14. Habermann TM, Weller EA, Morrison VA, Gascoyne RD, Cassileth PA, Cohn JB, et al. Rituximab-CHOP versus $\mathrm{CHOP}$ alone or with maintenance rituximab in older patients with diffuse large B-cell lymphoma. J Clin Oncol. 2006;24:3121-7.

15. Pfreundschuh $M$, Kuhnt $E$, Trümper L, Osterborg A, Trneny $M$, Shepherd $L$, et al. CHOP-like chemotherapy with or without rituximab in young patients with goodprognosis diffuse large-B-cell lymphoma: 6-year results of an open-label randomised study of the MabThera International Trial (MInT) Group. Lancet Oncol. 2011;12:1013-22.

16. Pfreundschuh $M$, Trümper $L$, Osterborg $A$, Pettengell $R$, Trneny $M$, Imrie $K$, et al. $\mathrm{CHOP}$-like chemotherapy plus rituximab versus $\mathrm{CHOP}$-like chemotherapy alone in young patients with good-prognosis diffuse large-B-cell lymphoma: a randomised controlled trial by the MabThera International Trial (MInT) Group. Lancet Oncol. 2006;7:379-91.

17. Miao Y, Medeiros LJ, Li Y, Li J, Young KH. Genetic alterations and their clinical implications in DLBCL. Nat Rev Clin Oncol. 2019;16:634-52.

18. Roschewski M, Staudt LM, Wilson WH. Diffuse large B-cell lymphoma-treatment approaches in the molecular era. Nat Rev Clin Oncol. 2014;11:12-23.

19. Sehn LH, Gascoyne RD. Diffuse large B-cell lymphoma: optimizing outcome in the context of clinical and biologic heterogeneity. Blood. 2015;125:22-32.

20. Goy A, Ramchandren R, Ghosh N, Munoz J, Morgan DS, Dang NH, et al. Ibrutinib plus lenalidomide and rituximab has promising activity in relapsed/refractory non-germinal center B-cell-like DLBCL. Blood. 2019;134:1024-36.

21. Wright G, Tan B, Rosenwald A, Hurt EH, Wiestner A, Staudt LM. A gene expression-based method to diagnose clinically distinct subgroups of diffuse large B cell lymphoma. Proc Natl Acad Sci USA. 2003;100:9991-6.

22. Lenz G, Wright G, Dave SS, Xiao W, Powell J, Zhao H, et al. Stromal gene signatures in large-B-cell lymphomas. N. Engl J Med. 2008;359:2313-23.

23. Petrich AM, Gandhi M, Jovanovic B, Castillo JJ, Rajguru S, Yang DT, et al. Impact of induction regimen and stem cell transplantation on outcomes in double-hit lymphoma: a multicenter retrospective analysis. Blood. 2014;124:2354-61.

24. Hu S, Xu-Monette ZY, Tzankov A, Green T, Wu L, Balasubramanyam A, et al. MYC/ $\mathrm{BCL} 2$ protein coexpression contributes to the inferior survival of activated B-cell subtype of diffuse large B-cell lymphoma and demonstrates high-risk gene expression signatures: a report from The International DLBCL Rituximab-CHOP Consortium Program. Blood. 2013;121:4021-31. quiz 4250

25. Horn H, Ziepert M, Becher C, Barth TFE, Bernd H-W, Feller AC, et al. MYC status in concert with $\mathrm{BCL} 2$ and $\mathrm{BCL} 6$ expression predicts outcome in diffuse large B-cell lymphoma. Blood. 2013;121:2253-63.

26. Green TM, Young KH, Visco C, Xu-Monette ZY, Orazi A, Go RS, et al. Immunohistochemical double-hit score is a strong predictor of outcome in patients with diffuse large B-cell lymphoma treated with rituximab plus cyclophosphamide, doxorubicin, vincristine, and prednisone. J Clin Oncol. 2012;30:3460-7.

27. Barraclough $A$, Alzahrani $M$, Ettrup MS, Bishton $M$, van Vliet $C$, Farinha $P$, et al. $\mathrm{COO}$ and $\mathrm{MYC/BCL2}$ status do not predict outcome among patients with stage $\mathrm{I} / \mathrm{II}$ DLBCL: a retrospective multicenter study. Blood Adv. 2019;3:2013-21.
28. Kumar A, Lunning MA, Zhang Z, Migliacci JC, Moskowitz $\mathrm{CH}$, Zelenetz AD Excellent outcomes and lack of prognostic impact of cell of origin for localized diffuse large B-cell lymphoma in the rituximab era. $\mathrm{Br} J$ Haematol. 2015;171:776-83.

29. Scott DW, Mottok A, Ennishi D, Wright GW, Farinha P, Ben-Neriah S, et al. Prognostic significance of diffuse large B-Cell lymphoma cell of origin determined by digital gene expression in formalin-fixed paraffin-embedded tissue biopsies. J Clin Oncol. 2015;33:2848-56.

30. Landsburg DJ, Falkiewicz MK, Maly J, Blum KA, Howlett C, Feldman T, et al. Outcomes of patients with double-hit lymphoma who achieve first complete remission. J Clin Oncol. 2017;35:2260-7.

31. Stephens DM, Li H, Persky D, Park SI, Bartlett NL, Swinnen LJ, et al. Characteristics and outcomes of patients with double-protein expression in limited stage diffuse large B-Cell lymphoma (DLBCL): analysis of SWOG study S1001 (NCT01359592). Blood. 2017;130:4122-4122.

32. Wang $Y$, Link BK, Witzig TE, Maurer MJ, Allmer C, King RL, et al. Impact of concurrent indolent lymphoma on the clinical outcome of newly diagnosed diffuse large B-cell lymphoma. Blood. 2019;134:1289-97.

33. Lacy SE, Barrans SL, Beer PA, Painter D, Smith AG, Roman E, et al. Targeted sequencing in DLBCL, molecular subtypes, and outcomes: a Haematological Malignancy Research Network report. Blood. 2020;135:1759-71.

34. Jones SE, Miller TP, Connors JM. Long-term follow-up and analysis for prognostic factors for patients with limited-stage diffuse large-cell lymphoma treated with initial chemotherapy with or without adjuvant radiotherapy. J Clin Oncol. 1989;7:1186-91.

35. Spicer J, Smith $\mathrm{P}$, Maclennan K, Hoskin $\mathrm{P}$, Hancock B, Linch D, et al. Long-term follow-up of patients treated with radiotherapy alone for early-stage histologically aggressive non-Hodgkin's lymphoma. Br J Cancer. 2004;90:1151-5.

36. Stephens DM, Li H, LeBlanc ML, Puvvada SD, Persky D, Friedberg JW, et al Continued risk of relapse independent of treatment modality in limited-stage diffuse large B-cell lymphoma: final and long-term analysis of Southwest Oncology Group Study S8736. J Clin Oncol. 2016;34:2997-3004.

37. Reyes F, Lepage E, Ganem G, Molina TJ, Brice P, Coiffier B, et al. ACVBP versus CHOP plus radiotherapy for localized aggressive lymphoma. N. Engl J Med. 2005:352:1197-205.

38. Bonnet C, Fillet G, Mounier N, Ganem G, Molina TJ, Thiéblemont C, et al. CHOP alone compared with $\mathrm{CHOP}$ plus radiotherapy for localized aggressive lymphoma in elderly patients: a study by the Groupe d'Etude des Lymphomes de l'Adulte. J Clin Oncol. 2007;25:787-92.

39. Persky DO, Unger JM, Spier CM, Stea B, LeBlanc M, McCarty MJ, et al. Phase II study of rituximab plus three cycles of $\mathrm{CHOP}$ and involved-field radiotherapy for patients with limited-stage aggressive B-cell lymphoma: Southwest Oncology Group study 0014. J Clin Oncol. 2008;26:2258-63.

40. Phan J, Mazloom A, Medeiros LJ, Zreik TG, Wogan C, Shihadeh F, et al. Benefit of consolidative radiation therapy in patients with diffuse large B-cell lymphoma treated with R-CHOP chemotherapy. J Clin Oncol. 2010;28:4170-6.

41. Pfreundschuh $M$, Murawski N, Ziepert $M$, Altmann B, Dreyling MH, Borchmann $P$, et al. Radiotherapy (RT) to bulky (B) and extralymphatic (E) disease in combination with $6 \mathrm{xR}-\mathrm{CHOP}-14$ or R-CHOP-21 in young good-prognosis DLBCL patients: results of the $2 \times 2$ randomized UNFOLDER trial of the DSHNHL/GLA. J Clin Orthod. 2018:36:7574-7574.

42. Elstrom RL, Leonard JP, Coleman M, Brown RKJ. Combined PET and low-dose, noncontrast CT scanning obviates the need for additional diagnostic contrastenhanced CT scans in patients undergoing staging or restaging for lymphoma. Ann Oncol. 2008;19:1770-3.

43. Cheah $C Y$, Hofman MS, Dickinson M, Wirth A, Westerman D, Harrison SJ, et al Limited role for surveillance PET-CT scanning in patients with diffuse large B-cell lymphoma in complete metabolic remission following primary therapy. $\mathrm{Br} J$ Cancer. 2013;109:312-7.

44. Cheung MC, Mittmann N, Earle CC, Rahman F, Liu N, Singh S. Are we choosing wisely in lymphoma? Excessive use of surveillance $C T$ imaging in patients with diffuse large B-cell Lymphoma (DLBCL) in long-term remission. Clin Lymphoma Myeloma Leuk. 2018;18:e27-34.

45. Sehn LH, Savage KJ, Hoskins P, Klasa R, Shenkier T, Gascoyne RD, et al. Treatment of limited-stage DLBCL can be effectively tailored using a PET-based approach. Annals of Oncology. 2011;22:90-1.

46. Sehn LH. Chemotherapy alone for localized diffuse large B-cell lymphoma. Cancer J. 2012;18:421-6.

47. S Bologna, T Vander Borght, J Briere, V Ribrag, GL Damaj, C Thieblemont, et al. Bastie. Early Positron Emission Tomography Response-Adapted Treatment in Localized Diffuse Large B-Cell Lymphoma (aalPI=0): Results of the Phase 3 LYSA LNH 09-1B Trial. Hematol Oncol. 2021. https://doi.org/10.1002/hon.5_2879.

48. Mikhaeel NG, Smith D, Dunn JT, Phillips M, Møller H, Fields PA, et al. Combination of baseline metabolic tumour volume and early response on PET/CT improves 
progression-free survival prediction in DLBCL. Eur J Nucl Med Mol Imaging. 2016;43:1209-19.

49. Zhao P, Yu T, Pan Z. Prognostic value of the baseline 18F-FDG PET/CT metabolic tumour volume (MTV) and further stratification in low-intermediate (L-I) and high-intermediate $(\mathrm{H}-\mathrm{I})$ risk NCCNIPI subgroup by MTV in DLBCL MTV predict prognosis in DLBCL. Ann Nucl Med. 2020. https://doi.org/10.1007/s12149-02001531-1.

50. Poeschel V, Held G, Ziepert M, Witzens-Harig M, Holte $H$, Thurner L, et al. Four versus six cycles of $\mathrm{CHOP}$ chemotherapy in combination with six applications of rituximab in patients with aggressive B-cell lymphoma with favourable prognosis (FLYER): a randomised, phase 3, non-inferiority trial. Lancet. 2020;394:2271-81.

51. Lugtenburg EJ, Brown $P$, van der Holt B, D'Amore F, Koene HR, de Jongh $E$, et al. Rituximab maintenance for patients with diffuse large B-cell lymphoma in first complete remission: results from a randomized HOVON-Nordic Lymphoma Group phase III study. J Clin Orthod. 2019;37:7507-7507.

52. Rozental A, Gafter-Gvili A, Vidal L, Raanani P, Gurion R. The role of maintenance therapy in patients with diffuse large B cell lymphoma: a systematic review and meta-analysis. Hematol Oncol. 2019;37:27-34.

53. Tokola S, Kuitunen H, Turpeenniemi-Hujanen T, Kuittinen O. Significance of bulky mass and residual tumor-Treated with or without consolidative radiotherapy-To the risk of relapse in DLBCL patients. Cancer Med. 2020;9:1966-77.

54. Grass GD, Mills MN, Ahmed KA, Liveringhouse CL, Montejo ME, Robinson TJ, et al. Radiotherapy for early stage diffuse large B-cell lymphoma with or without double or triple hit genetic alterations. Leuk Lymphoma. 2019;60:886-93.

55. Bobillo S, Joffe E, Lavery JA, Sermer D, Ghione P, Noy A, et al. Clinical characteristics and outcomes of extranodal stage I diffuse large $B$ cell lymphoma in the rituximab-era. Blood. 2020. https://doi.org/10.1182/blood.2020005112

56. Møller MB, Pedersen NT, Christensen BE. Diffuse large B-cell lymphoma: clinical implications of extranodal versus nodal presentation-a population-based study of 1575 cases. Br J Haematol. 2004;124:151-9.

57. Stephens DM, Li H, Constine LS, Leonard JP, Kahl BS, Song JY, et al. Extranodal presentation in limited stage DLBCL as a prognostic marker in three sequential SWOG trials S0014, S0313 and S1001 (NCT00005089, NCT00070018, NCT01359592). Blood. 2021;138:1423.

58. Nijland M, Boslooper K, van Imhoff G, Kibbelaar R, Joosten P, Storm H, et al. Relapse in stage I(E) diffuse large B-cell lymphoma. Hematol Oncol. 2018;36:416-21.

59. Kridel R, Telio D, Villa D, Sehn LH, Gerrie AS, Shenkier T, et al. Diffuse large B-cell lymphoma with testicular involvement: outcome and risk of CNS relapse in the rituximab era. Br J Haematol. 2017;176:210-21.

60. Vitolo U, Chiappella A, Ferreri AJM, Martelli M, Baldi I, Balzarotti M, et al. First-line treatment for primary testicular diffuse large B-cell lymphoma with rituximabCHOP, CNS prophylaxis, and contralateral testis irradiation: final results of an international phase II trial. J Clin Oncol. 2011;29:2766-72.

61. Munoz J, Kuriakose P. Antibiotic-refractory sinusitis. JAMA. 2012;308:2399-2400.

62. Orellana-Noia VM, Reed D, McCook AA, Sen JM, Barlow CM, Malecek M-K, et al. Single-route CNS prophylaxis for aggressive non-Hodgkin lymphomas: real-world outcomes from 21 US academic institutions. Blood. 2021. https://doi.org/10.1182/ blood.2021012888.

63. Hollender A, Kvaloy S, Nome O, Skovlund E, Lote K, Holte H. Central nervous system involvement following diagnosis of non-Hodgkin's lymphoma: a risk model. Ann Oncol. 2002;13:1099-107.

64. Tai WM, Chung J, Tang PL, Koo YX, Hou X, Tay KW, et al. Central nervous system (CNS) relapse in diffuse large $B$ cell lymphoma (DLBCL): pre- and post-rituximab. Ann Hematol. 2011;90:809-18.
65. Schmitz C, Rekowski J, Müller SP, Farsijani N, Hertenstein B, Franzius C, et al. Impact of complete surgical resection on outcome in aggressive non-Hodgkin lymphoma treated with immunochemotherapy. Cancer Med. 2020;9:8386-96.

66. Yoon DH, Sohn BS, Oh SY, Lee W-S, Lee SM, Yang D-H, et al. Feasibility of abbreviated cycles of immunochemotherapy for completely resected limited-stage CD20+ diffuse large B-cell lymphoma (CISL 12-09). Oncotarget. 2017;8:13367-74.

67. Kang S, Cho H, Sohn BS, Oh SY, Lee W-S, Lee SM, et al. Long-term follow-up of abbreviated R-CHOP chemoimmunotherapy for completely resected limitedstage diffuse large B cell lymphoma (CISL 12-09). Ann Hematol. 2020;99:2831-6.

68. Dührsen U, Müller S, Hertenstein B, Thomssen $H$, Kotzerke J, Mesters R, et al. Positron emission tomography-guided therapy of aggressive non-Hodgkin lymphomas (PETAL): a multicenter, randomized phase III Trial. J Clin Oncol. 2018;36:2024-34.

69. Kim SJ, Kang HJ, Kim JS, Oh SY, Choi CW, Lee Sl, et al. Comparison of treatment strategies for patients with intestinal diffuse large B-cell lymphoma: surgical resection followed by chemotherapy versus chemotherapy alone. Blood. 2011;117:1958-65.

\section{AUTHOR CONTRIBUTIONS}

AER and SMS designed, wrote, edited, and approve the final version of this manuscript.

\section{COMPETING INTERESTS}

A.E.R. has no competing interests to report. S.M.S. has no competing interests related to this work. There was no funding from any sources for this work. Per ICJME guidelines, S.M.S. has served as a consultant for ADC Therapeutics, Gilead, BMS, Morphosys, Janssen, Karyopharm, Genentech, TGTX, Celgene, Bayer.

\section{ADDITIONAL INFORMATION}

Correspondence and requests for materials should be addressed to Sonali M. Smith.

Reprints and permission information is available at http://www.nature.com/ reprints

Publisher's note Springer Nature remains neutral with regard to jurisdictional claims in published maps and institutional affiliations.

Open Access This article is licensed under a Creative Commons Attribution 4.0 International License, which permits use, sharing, adaptation, distribution and reproduction in any medium or format, as long as you give appropriate credit to the original author(s) and the source, provide a link to the Creative Commons license, and indicate if changes were made. The images or other third party material in this article are included in the article's Creative Commons license, unless indicated otherwise in a credit line to the material. If material is not included in the article's Creative Commons license and your intended use is not permitted by statutory regulation or exceeds the permitted use, you will need to obtain permission directly from the copyright holder. To view a copy of this license, visit http://creativecommons. org/licenses/by/4.0/.

(c) The Author(s) 2022 